\title{
QUANTUM EFFICIENCY OF AN INVERSION LAYER SILICON PHOTODIODE IN THE NEAR ULTRAVIOLET
}

\author{
M. S. Khalil and M.El-Sherif \\ National Institute for Standards, Giza, Egypt
}

\begin{abstract}
The potential of an available oxide $-n^{*}-p$ inversion layer silicon photodiode as a standard radiometric detector in the near ultraviolet (250-400 nm) has been discussed. High accuracy measurements of the quantum efficiency have been performed using self calibration technique. It was found that applying a reverse bias voltage extends the quantum efficiency over the ultraviolet region. The results due to various radiant levels are given. Radiant energy measured with this diode was compared with that measured by an electrically calibrated pyrometer. The examined detector showed stable characteristics with time and therefore it is considered ideal for radiation measurements in the near ultraviolet.
\end{abstract}

\section{Introduction}

A radiometric standard scale in visible region has been recently established at the National Institute for Standards in Egypt [1]. The scale was based on accurately characterized inversion layer photodiodes This type of photodiode was developed by Hansen [2] and manufactured by United Detector Technology in USA. It has thermally grown silicon dioxide layer on the front surface acting as antireflection coating. The junction is induced in the silicon by positive charges trapped in the silicon oxide.

To extend the radiometric scale in the ultraviolet, more investigations were carried out to characterize the photodiode in this region. The external quantum efficiency of the photodiode at a certain wavelength can be determined using a self calibration technique [3]. This is based on the determination of the parameters describing the recombination mechanism in the diode and the measurement of the 
surface reflectance.

The main problem in this technique is the measurement of losses occurring in the device. A physical model has been used to describe the reduction in the quantum efficiency of the inversion layer photodiode. Using this technique, several measurements with zero bias, oxide bias and reverse bias were performed to determine the relative losses which decrease the quantum efficiency of the photodiode to less than unity [4].

The photodiode's absolute spectral response $S(\lambda)$ at a wavelength $\lambda$ is calculated from its internal quantum efficiency $\varepsilon(\lambda)$ using the relation:

$$
S(\lambda)=(1-\rho(\lambda)) \cdot \varepsilon(\lambda) \text { e } \lambda / \text { hc }
$$

where

$\rho(\lambda)$ is the reflectance of the front surface of the photodiode,

e is the elementary electron charge.

$\mathrm{h}$ is the Planck's constant and

c is the velocity of light.

The diode internal quantum efficiency $\varepsilon$ in equation (1) is given by :

$$
\varepsilon(\lambda)=\varepsilon_{0}(\lambda) \cdot \varepsilon_{k}(\lambda) \cdot\left[1-\left(1-\varepsilon_{0}(\lambda)\right)\left(1-\varepsilon_{k}(\lambda)\right)\right]^{-1} .
$$

$\varepsilon_{\mathrm{o}}(\lambda)$ is defined as the ratio of the photocurrent obtained with zero bias voltage across the oxide to that obtained with a bias voltage sufficient to saturate the photocurrent. The quantity $\varepsilon_{k}(\lambda)$ is defined in a similar way in terms of the reverse bias voltage.

\section{Reflectance measurements :}

Since the front surface of the photodiode is of optical quality, the reflected radiation can be determined by specular measurements. The photodiode was packaged with two other diodes of the same type on a black frame to form a light-trapping device as shown in Fig. (1). More details on the design and construction of the device has been illustrated in reference [1]. A circular aperture was attached to the front of the frame. The light coming through the aperture strikes the first at 45 degree incidence. Most of the light is absorbed at this surface. The reflected light is directed to the second detector. Again most of light is absorbed and the remainder directed to the third 
detector. Any remaining light from the third detector is reflected back on itself to traverse the path towards the aperture. So, the light has to make five specular reflections before any is lost.

The signal from each detector was available for measurement in addition to the electrical signal for the sum of all three detectors. This also allowed signals to be measured at different radiant power levels at one setting, by detecting the signal produced by detectors 1,2 and 3 .

The reflectance from the first detector was measured by blocking the radiation from reaching the other two detectors and measuring the current $I_{1}$. Then by unblocking the beam, the photocurrent from all three photodiodes $\mathrm{I}_{2}$ was measured. If the differences between 45 and 0 degree incidence is neglected, the reflectance $\rho$, can be approxiamted as:

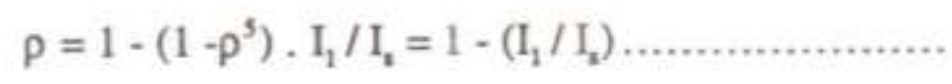

At other normal incidence, the reflectance of the diode is sensitive to polarization in the ultraviolet. To minimize the sensitivity of the package to any non-uniform polarization in the incident beam, the detectors were arranged so that they rotates the plane of incidence 90 degrees on each reflection. The two planes of polarization of the incident beam therefore alternate between high and low reflectance at successive surfaces.

Fig. (2) shows the reflectance measurements of the photodiode at TE, TM and unpolarized radiation for five wavelengths in the ultraviolet. The experimental data has been best fitted using the least squares method to give the reflectance of the diode at any intermediate wavelength in the considered range. The curves are well fitted by a polynomial of the form:

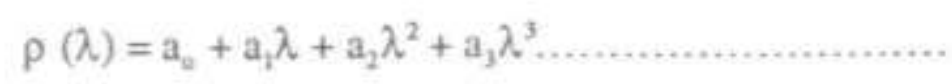

The coefficients $a_{6}, a_{1}$ and $a_{2}, a$, for the unpolarized radiation curve are found $0.595,9.09 \times 10^{-4},-1.05 \times 10^{-5}$ and $1.86 \times 10^{-4}$. respectively. 


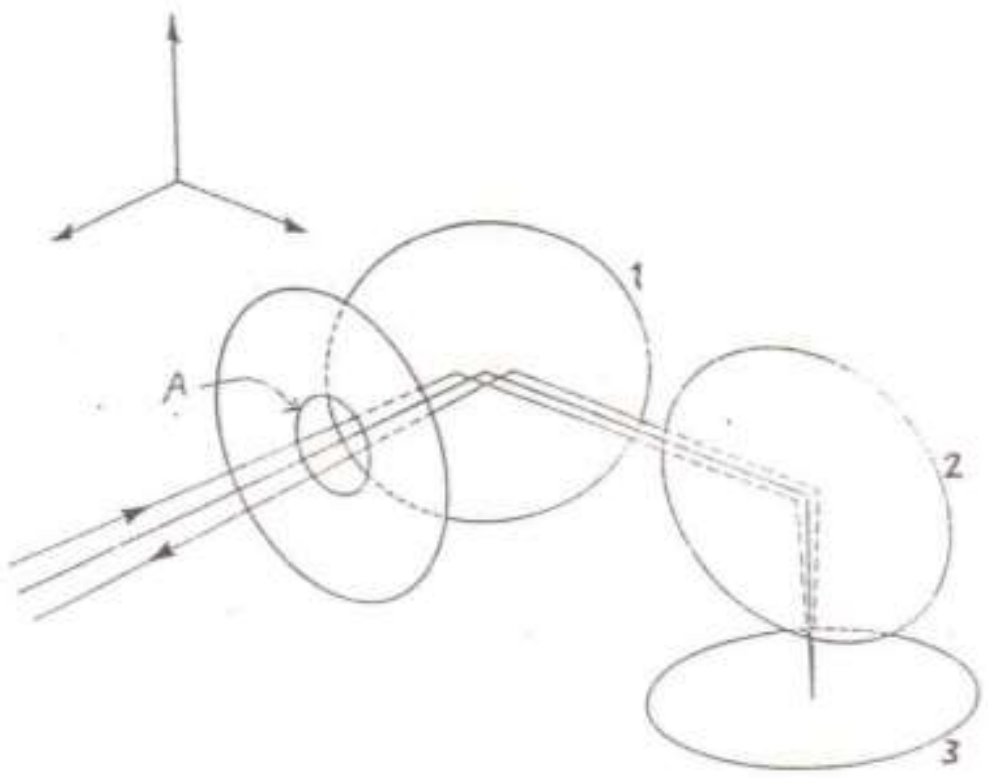

FIG.1 Arrangment of the three photodiodes in the package.

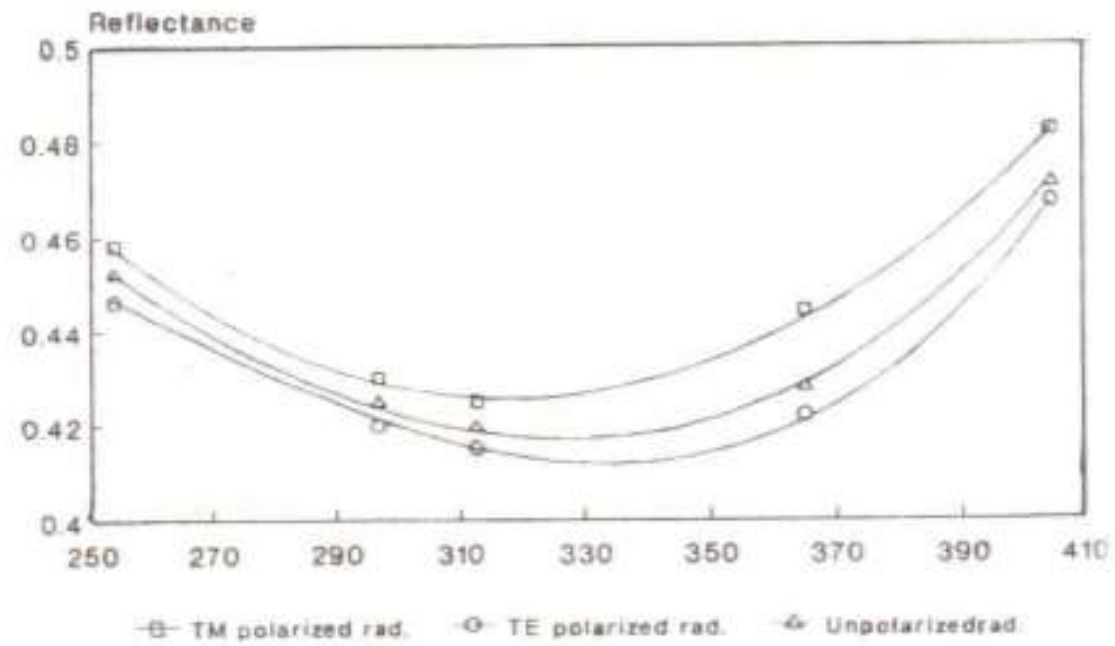

FIG 2. Reflectance of the diode at TM, EM and unpolarized radiation. 
For the TM polarized curve the coefficients are $1.23,-5.18 \times 10^{-3}$, $8.66 \times 10^{-3},-1.07 \times 10^{-5}$, while that for TE polarized curve are $-0.052,6.97 \times 10^{-3},-2.93 \times 10^{-5}, 3.76 \times 10^{-5}$. The reflection differences between the two planes of polarization and the unpolarized radiation are too small to affect the calculated external quantum efficiency of the diode at the ultraviolet range.

\section{Reverse bias measurements}

If a voltage is applied so as to reverse bias the photodiode, the charge carriers are moved out of the depletion region before they have had chance to recombine and therefore the output signal is raised $[5,6]$. As the bias voltage increases, the output current reaches maximum, called saturation current and that means the collection of all electron-hole pairs. To estimate the quantum efficiency from equation (1), $\varepsilon$ is obtained as the ratio of the photocurrent with zero bias voltage across the oxide to that obtained with a bias voltage sufficient to saturate the photocurrent. Fig. (3) shows the behavior of the output signal at three wavelengths in the ultraviolet as the reverse bias is increased. It is shown that the maximum percentage increase, as the voltage raises from zero bias to 30 Volts occurs at the wavelength $365 \mathrm{~nm}$. This amount decreases at shorter wavelengths which means that the effect of residual recombination of electron-hole pairs is negligible. As the reverse bias voltage was increased, the noise in the measured signal was noticed to increase with the signal itself. At shorter wavelengths the signal was getting smaller, so it is recommended that the bias voltage does not exceed $30 \mathrm{~V}$.

\section{Source of ultraviolet radiation}

The source of ultraviolet was a high pressure mercury vapour lamp of 400 watts The glass envelope of the lamp has been removed to permit emission of the ultraviolet through the inner quartz bulb. A double monochromator has been used with the lamp to give monochromatic radiation through the exist slit and directed to the photodiode. The measurements of the ultraviolet radiation were carried out using the high intensity lines of mercury 253.7, 296.8, $312.6,365$ and $404.6 \mathrm{~nm}$. 


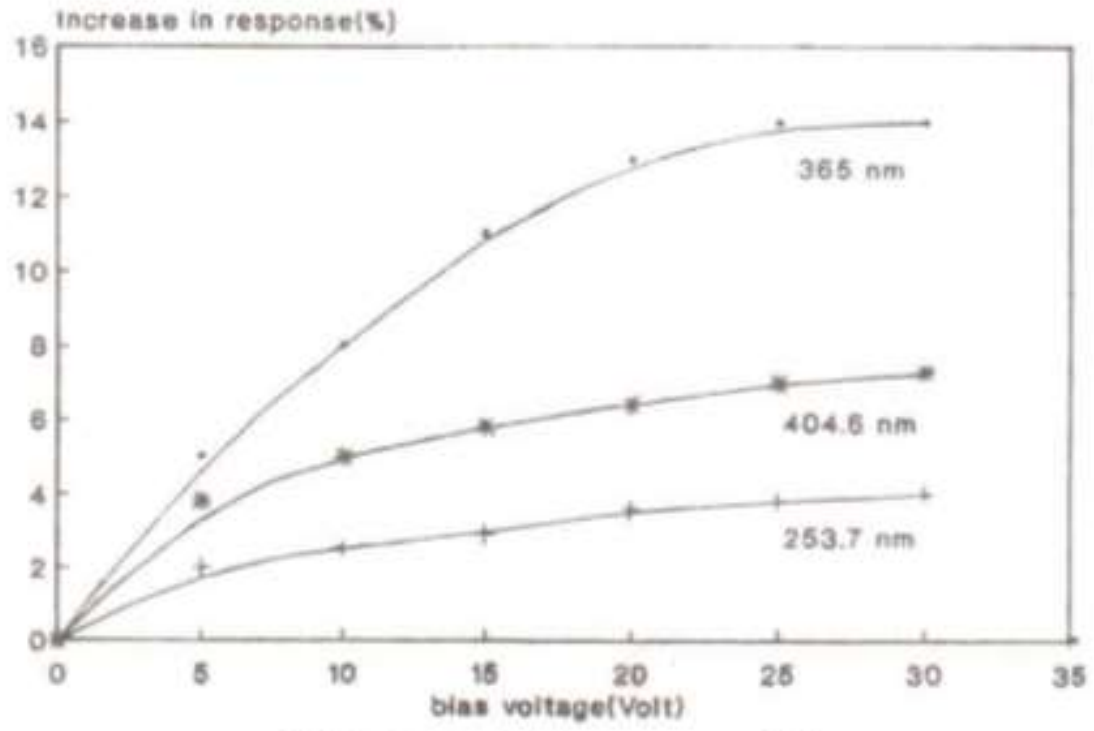

FIG.3. Increase of response of the photodiode with bias voltage.

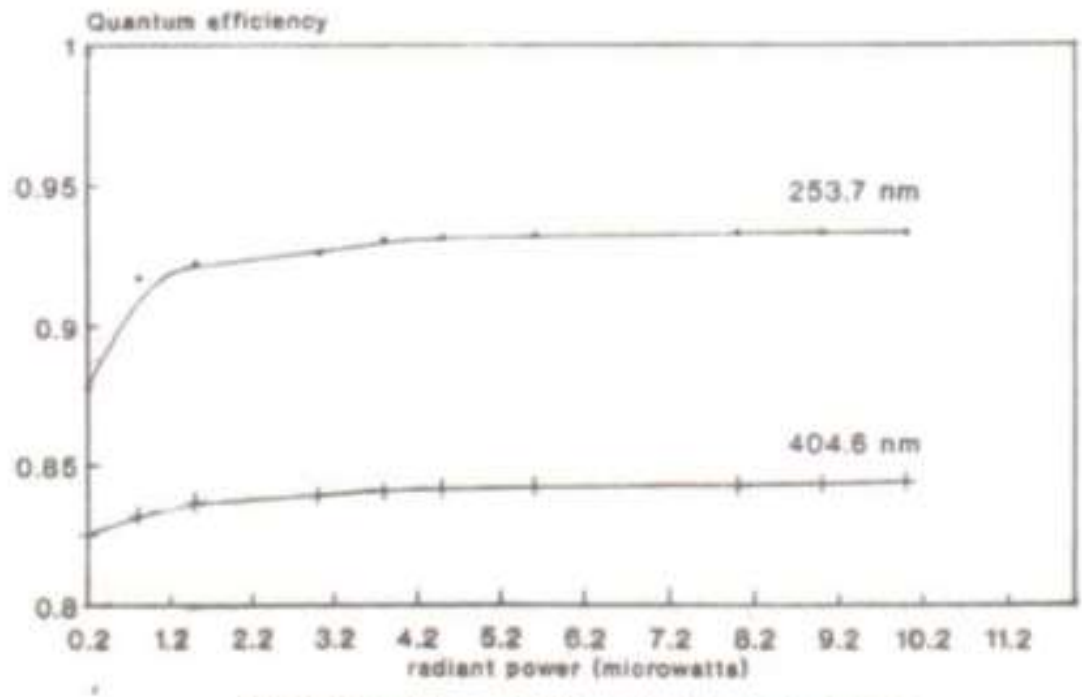

FIG.4. Quantum efficiency of the diode as a function of radiant power at the wavelengthe 253.7 and $404.6 \mathrm{~nm}$. 


\section{Pyroelectric radiometer}

An electrically calibrated pyroelectric radiometer Rs-5900 was used as the reference instrument for the radiation power. The pyrometer has been calibrated in ultraviolet at National Bureau of Standards (USA). The detector head consists of a lithium tantalate with a thin film heater deposited on its surface [7]. The radiant power is directly compared to the measurement of electrical power dissipated within the detector itself.

\section{Results and discussion}

The results of the external quantum efficiency measurements of the inversion layer photodiode is given in Table (1). The table gives the values of the reflectance $\rho$, internal quantum efficiency $\varepsilon$ and external quantum efficiency $S$ in amperes/watt for five wavelengths. It is shown that the internal quantum efficiency increases by shorter wavelengths. The results of intercomparison with the electrical pyrometer indicates a maximum difference of $4.2 \%$ at $253.7 \mathrm{~nm}$. This significant error was attributed to the small power level which produces a small signal at this wavelength. It may also due to the effect of impact ionization on the photodiode which increases at short wavelengths.

Over $300 \mathrm{~nm}$, the difference is $<2 \%$. Fig. (4) shows the measured quantum efficiency as a function of radiant power at the wavelengths $253.7 \mathrm{~nm}$ and $404.6 \mathrm{~nm}$ at zero bias. At low powers the quantum efficiency is relatively low. So, it is necessary to apply a reverse bias voltage to get the maximum quantum efficiency.

\section{Conclusion}

The method described in this paper facilitates the frequent realization of the spectral irradiance scale in the ultraviolet. It can be used in any moderately equipped laboratory to establish an independent standard scale in the ultraviolet. The absolute spectral response (external quantum efficiency) of an inversion layer silicon photodiode has been obtained in this region from reflectance 
measurements and the internal quantum efficiency values of the diode. The internal quantum efficiency has been deduced from measurements of the increase in response as a function of bias voltage.

Table (I): Absolute spectral response and comparison of selfcalibrated silicon photodiode with an electrically calibrated pyrometer.

\begin{tabular}{|c|c|c|c|c|}
\hline Wavelength & Reflectance $(\varphi)$ & Quantum eff. $(\rho)$ & $S$ (Amp/Watt) & Silicon/Pyrometer \\
\hline 235.7 & 0.435 & 0.9327 & 0.1064 & 0.9579 \\
\hline 296.8 & 0.4535 & 0.9624 & 0.1302 & 0.9746 \\
\hline 312.6 & 0.418 & 0.9316 & 0.1367 & 0.9826 \\
\hline 365 & 0.413 & 0.8436 & 0.1458 & 0.9875 \\
\hline 404.6 & 0.473 & 0.8864 & 0.1524 & 0.9881 \\
\hline
\end{tabular}

\section{References :}

1) M. Shafik Khalil, and M. M. Khodair, Proc, Egypt. Acid. Sci, 36, 85 (1986/1987).

2) T. E. Hansen,Phys. Scr., 18, 471 (1978).

3) Robert 1. Booker and John Geist, App. Opt.12, 1940, (1984).

4) John Geist, Eli Liang, and A. Russell Schaefer, Appl. Phys.52, 4897 (1981).

5) Edward F, Zalewsi and C. Richard Duda," App. Opti., 18, 2867, (1983).

6) J. Geist, E. F. Zalewski, and A. R. Schaefer, Appl. Opt. 19, 3975-3798.

7) C. A. Hamilton, G, A. day, R. J. Phelan, NBS (USA). Tech. Note 678 (March 1976). 